\title{
WATER QUALITY INDEX OF CHITRAPUZHA RIVER, ERNAMKULAM, KERALA, INDIA
}

\author{
G. DEEPA ${ }^{1} \&$ P. N. MAGUDESW ARAN ${ }^{2}$ \\ ${ }^{1}$ Research and Development Centre, Bharatiar University, Coimbatore, Tamil Nadu, India \\ ${ }^{2}$ Department of Chemistry, Hindustan College of Engineering and Technology, Coimbatore, Tamilnadu, India
}

\begin{abstract}
The present investigation was carried out for determining the various physico-chemical parameters and biological characteristic of Chitrapuzha River for two different seasons rainy and summer in Ernamkulam district. The following parameters were analyzed DO, FC, pH, BOD, Phosphate, Nitrates, Turbidity and TDS. All the measured parameters were found to be very high compared to limit prescribed by WHO, and thereby unfit for both drinking and irrigation. The Chitrapu zha River has been falling severe anthropogenic activities mostly due to industrial wastes.
\end{abstract}

KEYWORDS: Physico-Chemical Parameters, WHO Standard, Chitrapuzha River

\section{INTRODUCTION}

Chitrapuha river, one of the tributaries of Periyar river, flows through Amabalamedu, Kochi area, on the southern coast of Indian subcontinent. The river receives a variety of effluents from fertilizer, refinery and other industries. Apart from Fertilizers And Chemicals Travancore (FACT) other major industries around Ambalamedu Kochi area are Hindustan Organics Chemicals Limited (HOCL) and Kochi Refinery Limited (KRL). The effluents contain ammonia, ammonium sulphate, phos phate, calciu m sulphate, nitrate and heavy metals

Effluents from these industrial units along with agricultural and other anthropogenic effluents find their way into Chitrapuzha River ultimately into Cochin backwaters. There are long standing local complaints about water pollution causing fish mortality and serious damage to agricultural crops resulting in extensive unemploy ment in the area. The lower reaches of this river became part of National Waterways in 1993 and is now mainly used for transporting chemicals from Cochin Port to the industrial units located on the banks of the river. The total effluent discharge into Chitrapuzha river is about 33,600 m3 per day. This study helps us to assess the impacts of industrial effluents and domestic sewage on surface water quality of Chitrapuzha river

\section{MATERIALS AND METHODS}

Based on specific geographical features, water flow regimes and anthropogenic activities, 9 sampling locations were selected (Figure 1). The samples were collected in acid-washed 5 liter plastic bottles at 10 a.m. every three days, continuously for one month period during rainy and summer season. For chemical, biological and microbiological examination, different methods of collection and handling were adopted. The instruments were used of precise accuracy and chemicals used were of AR grade.

PH was measured using Digital pH meter. DO and BOD was measured using Winkler's titrimetric method. The multiple-tube fermentation method was used to determine the bacteria present. The confirmed and complete test was carried out for the samples by using the nutrient froth. The turbidity was measured by using Digital Turbid ity meter, 863D 'Bio-Chem make. The evaporation method is used for determining the total solids by using standard procedures. Nitrate ion was determined using Brucine method. To determine the total phosphorus stannous chloride method is used by following the established procedure. 


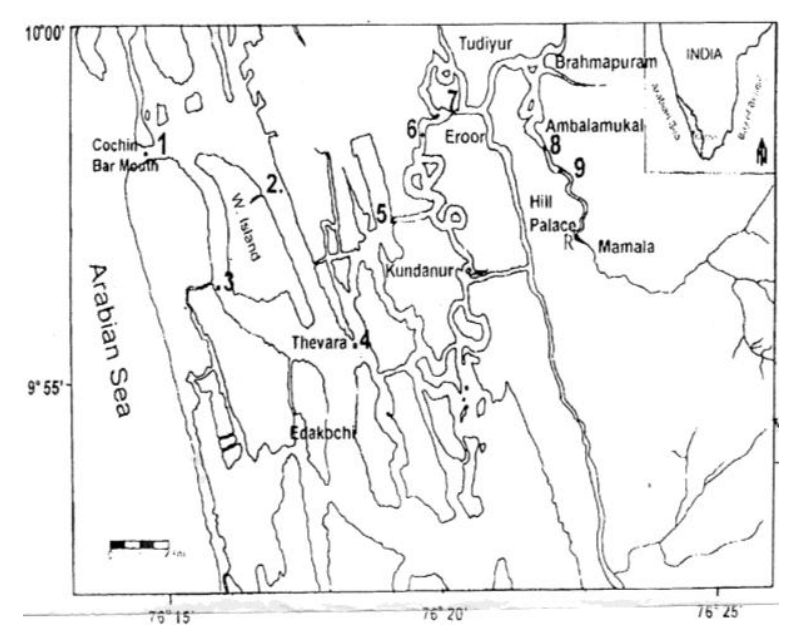

Figure 1: Sampling Stations in Chithrapuzha River

\section{RESULT AND DISCUSSIONS}

The physico-chemical and biological characteristics of the samples are given in table 1to9 for summer and rainy season along with the respective WQI value. The water quality index was calculated using the eight parameters (Magudeswaran 2004).

The eight resulting values were then added to an overall WQI (Magudeswaren, 2004). WQI $=0.19 \mathrm{DO}+0.18 \mathrm{FC}$ $+0.12 \mathrm{pH}+0.12 \mathrm{BOD}+0.11$ Total phosphate +0.11 Nitrates +0.09 Tu rbidity +0.08 Total solids .

Dissolved oxygen plays an important role in water quality determination. The study was that, the DO of river water was maximum at station 1 and less at station 9. The DO \% saturation of the river water at station 1 and 9 are 95.4 and 92 (Table 1\&9) for summer season and 98.9 and 91.50 for rainy season respectively.

Both in summer and rainy season the DO\% saturation was low at station 9 compared to station 1 . This due to addition of domestic sewage, industrial sewage and MSW containing oxidisable organic matter and consequent biodegradation and decay of vegetation, which leads to consumption of oxygen present in water (Jammel 1998). Low \% of saturation of DO has direct effect to fish community, especially during spawning period because the respiratory system requires DO to breath.

$\mathrm{pH}$ is a measurement of the acidity or basic quality of water. The $\mathrm{pH}$ of natural water is usually between 6.7 and 8.2. It was found that the $\mathrm{pH}$ of water varies from 6.7 to 8.5 in summer and 6.9 to 8.2 in rainy season (table 1 \& 9) showing that the alkalinity of water has increased. The Total solids are important parameters for drinking water and to be used for other purposes. According to WHO the permissible limits of total solids for drinking water is $1500 \mathrm{mg} / \mathrm{l}$ but the value of Chithrapuzha River water in station 9 exceeds this value due to mixing of sewage and industrial wastes.

BOD was low at station 1 and higher at station 9 during rainy and summer seasons. Desirable limit for BOD is $4.0 \mathrm{mg} / \mathrm{l}$ and permissible limit is $6.0 \mathrm{mg} / \mathrm{l}$ according to Indian standards. BOD below $3 \mathrm{mg} / \mathrm{l}$ or less is required for the best use. Fokmare and Musaddiq (2002) recorded high value of biochemical oxygen demand as $20.00 \mathrm{mg} / \mathrm{l}$ in river puma and said that this river is highly polluted due to organic enrichment, decay of plants and animal matter in the river. Thus the high value of BOD encountered in station 9 (table 9) during summer and rainyseason, above the permissible limit of WHO $(<2 \mathrm{mg} / \mathrm{l})$ indicates the pollution by biochemical degradable organic waste from various sources. 
Faecal coliform bacteria are living organisms, unlike the other conventional water quality parameters. Fecal coliforms are around $13 \mathrm{MPN} / 100 \mathrm{ml}$ at station 1, starts to rise and reaches the value $26.2 \mathrm{MPN} / 100 \mathrm{ml}$ in summer season at station 6 (table 6). The rising of Faecal coliform is the direct evidence for mixing of untreated sewage, poorly maintained septic systems, and scooped pet waste into the river water.

Turbidity and water colour can be regarded as aesthetic pollutants. The observed results are presented in tables 1 to 9. The high turbidity value of 11.2NTU was observed in station 9 during summer season (Table 9). The high content of turbidity station 9 may be due to increase in TDS value or it may be due to organic compounds being introduced in to it either through domestic or location area effluents (Agarwal, 2005). The concentration of phosphate in Chitrapuzha River was found to be ranged from $0.06 \mathrm{mg} / 1$ to $1.45 \mathrm{mg} / \mathrm{l}$ in summer and.03 to 1.32 in rainy season. Among the nine sampling stations, the station 9 during both rainy and summer seas on showed high phosphate content when compared to station 1. Phosphate is the indication of pollution by detergents and it leads to formation of algal bloom. The high concentration of phosphate in station 9 (Table 9) may be due to human and animal waste are flushed in to water ways, either from poorly treated sewage, surface runoff and some Industrial waste also carry phosphorous in to the river. The unfiltered water of the catchment area of phosphate rocks, uncontrolled disposal of sewage and biodegradable synthetic detergents also add huge quantities of phosphate (Agarwal, 2005).

The WQ index has decreased from station 1 to 9 by about 20.09 units (Tables 1\&9) in summer season and by about 14.38 units (Tables 1\&9) in rainy season. According to WQI legend the water which has the quality characteristics value around 25 is very bad and cannot be used for any purpose.

\section{CONCLUSIONS}

A WQI value of the chithrapuzha river (station 8,9) is not suitable for domestic and agricultural purposes as per drinking water standards of Bureau of Indian Standards and WHO guidelines. Regular monitoring of river and taking suitable remedial measures like collection of domestic sewage and setting up the common treatment plant before discharge of sewage in to river system is required. This will control pollution and prevent the depletion of the quality of Chitra Puzha River water.

Table 1: Calculation and Results of Water Quality Index (WQI)

\begin{tabular}{|c|c|c|c|c|c|c|}
\hline \multicolumn{7}{|c|}{ Station 1- Cochin Bar Mouth Summer 2013 } \\
\hline SI No & Parameters & Results & Units & Q Value & $\begin{array}{c}\text { Weighing } \\
\text { Factor( W) }\end{array}$ & $\begin{array}{c}\text { Sub } \\
\text { Total(QXW) }\end{array}$ \\
\hline 1 & DO & 98.2 & \% SATURATION & 99 & 0.19 & 18.81 \\
\hline 2 & FC & 13 & MPN/100 ML & 68 & 0.18 & 12.24 \\
\hline 3 & PH & 6.7 & PH UNITS & 79 & 0.12 & 9.48 \\
\hline 4 & BOD & 0.94 & MG/L & 95 & 0.12 & 11.4 \\
\hline 5 & P & 0.06 & NTU & 98 & 0.11 & 10.78 \\
\hline 6 & N & 1.08 & MG/L & 96 & 0.11 & 10.56 \\
\hline 7 & TURIDITY & 2.24 & NTU & 92 & 0.09 & 8.28 \\
\hline 8 & TDS & 149 & MG/L & 79 & 0.08 & 6.32 \\
\hline
\end{tabular}

Table 2: Calculation and Results of Water Quality Index (WQI)

\begin{tabular}{|c|c|c|c|c|c|c|}
\hline \multicolumn{7}{|c|}{ Station 2- Oil Tanker Berth Summer 2013 } \\
\hline SI No & Parameters & Results & Units & Q Value & $\begin{array}{c}\text { Weighing } \\
\text { Factor( W) }\end{array}$ & Sub Total (QXW) \\
\hline 1 & DO & 98.02 & \% SATURATION & 99 & 0.19 & 18.81 \\
\hline 2 & FC & 13.6 & MPN/100 ML & 68 & 0.18 & 12.24 \\
\hline
\end{tabular}




\begin{tabular}{l|c|c|c|c|c|c|}
\hline \multicolumn{7}{c|}{ Table 2: Contd., } \\
\hline 3 & PH & 6.7 & PH UNITS & 79 & 0.12 & 9.48 \\
\hline 4 & BOD & 0.98 & MG/L & 95 & 0.12 & 11.4 \\
\hline 5 & P & 0.09 & NTU & 96 & 0.11 & 10.56 \\
\hline 6 & N & 1.02 & MG/L & 96 & 0.11 & 10.56 \\
\hline 7 & TURBIDITY & 2.86 & NTU & 90 & 0.09 & 8.1 \\
\hline 8 & TDS & 150 & MG/L & 79 & 0.08 & 6.32 \\
\hline & & & & & \multicolumn{1}{c|}{ OVER ALL WQI=87.47 } \\
\hline
\end{tabular}

Table 3: Calculation and Results of Water Quality Index (WQI)

\begin{tabular}{|c|c|c|c|c|c|c|}
\hline \multicolumn{7}{|c|}{ Station 3- Thoppumpady Fishing Harbour Summer 2013 } \\
\hline SI No & Parame ters & Results & Units & Q Value & $\begin{array}{c}\text { Weighing } \\
\text { Factor }(\mathbf{W})\end{array}$ & Sub Total (QXW) \\
\hline 1 & DO & 98 & \% SATURATION & 99 & 0.19 & 18.81 \\
\hline 2 & FC & 16.2 & MPN/100 ML & 66 & 0.18 & 11.88 \\
\hline 3 & PH & 6.9 & PH UNITS & 86 & 0.12 & 10.32 \\
\hline 4 & BOD & 1.02 & MG/L & 95 & 0.12 & 11.4 \\
\hline 5 & P & 1 & NTU & 40 & 0.11 & 0.4 \\
\hline 6 & N & 1.65 & MG/L & 95 & 0.11 & 8.1 \\
\hline 7 & TURIDITY & 3.2 & NTU & 90 & 0.09 & 6.32 \\
\hline 8 & TDS & 152 & MG/L & 79 & 0.08 & OVER ALL WQI=81.68 \\
\hline
\end{tabular}

Table 4: Calculation and Results of Water Quality Index (WQI)

\begin{tabular}{|c|c|c|c|c|c|c|}
\hline \multicolumn{7}{|c|}{ Station 4- The vara Ferry Point Summer 2013 } \\
\hline SI No & Parame ters & Results & Units & Q Value & $\begin{array}{c}\text { Weighing } \\
\text { Factor ( W) }\end{array}$ & Sub Total (QXW) \\
\hline 1 & DO & 96.1 & \% SATURATION & 99 & 0.19 & 18.81 \\
\hline 2 & FC & 16.8 & MPN/100 ML & 65 & 0.18 & 11.7 \\
\hline 3 & PH & 7.1 & PH UNITS & 90 & 0.12 & 10.8 \\
\hline 4 & BOD & 1.25 & MG/L & 93 & 0.12 & 11.16 \\
\hline 5 & P & 1.04 & NTU & 39 & 0.11 & 4.29 \\
\hline 6 & N & 1.78 & MG/L & 95 & 0.11 & 10.45 \\
\hline 7 & TURIDITY & 3.62 & NTU & 89 & 0.09 & 8.01 \\
\hline 8 & TDS & 150 & MG/L & 79 & 0.08 & 6.32 \\
\hline & & & & & OVER ALL WQI=81.54 \\
\hline
\end{tabular}

Table 5: Calculation and Results of Water Quality Index (WQI)

\begin{tabular}{|c|c|c|c|c|c|c|}
\hline \multicolumn{7}{|c|}{ Station 5- Thykoodam Nh Bridge Summer 2013 } \\
\hline SI No & Parame ters & Results & Units & Q Value & $\begin{array}{c}\text { Weighing } \\
\text { Factor (W) }\end{array}$ & Sub Total (QXW) \\
\hline 1 & DO & 96.02 & \% SATURATION & 99 & 0.19 & 18.81 \\
\hline 2 & FC & 16.9 & MPN/100 ML & 65 & 0.18 & 11.7 \\
\hline 3 & PH & 7.2 & PH UNITS & 92 & 0.12 & 11.04 \\
\hline 4 & BOD & 1.56 & MG/L & 89 & 0.12 & 10.68 \\
\hline 5 & P & 1.03 & NTU & 39 & 0.11 & 4.29 \\
\hline 6 & N & 1.75 & MG/L & 95 & 0.11 & 10.45 \\
\hline 7 & TURIDITY & 3.8 & NTU & 88 & 0.09 & 7.92 \\
\hline 8 & TDS & 153 & MG/L & 79 & 0.08 & 6.32 \\
\hline
\end{tabular}

Table 6: Calculation and Results of Water Quality Index (WQI)

\begin{tabular}{|c|c|c|c|c|c|c|}
\hline \multicolumn{7}{|c|}{ Station 6- Kaniyampuzha RI wy Bridge Summer 2013 } \\
\hline SI No & Parame ters & Results & Units & Q Value & $\begin{array}{c}\text { Weighing } \\
\text { Factor( W) }\end{array}$ & Sub Total (QXW) \\
\hline 1 & DO & 95.4 & \% SATURATION & 98 & 0.19 & 18.62 \\
\hline
\end{tabular}




\begin{tabular}{|c|c|c|c|c|c|c|}
\hline \multicolumn{7}{|c|}{ Table 6: Contd., } \\
\hline 2 & $\overline{\mathrm{FC}}$ & 26.2 & MPN/100 ML & 60 & 0.18 & 10.8 \\
\hline 3 & $\mathrm{PH}$ & 8.3 & PH UNITS & 73 & 0.12 & 8.76 \\
\hline 4 & BOD & 2.34 & $\mathrm{MG} / \mathrm{L}$ & 73 & 0.12 & 8.76 \\
\hline 5 & $\mathrm{P}$ & 1.38 & NTU & 33 & 0.11 & 3.63 \\
\hline 6 & $\mathrm{~N}$ & 1.78 & $\mathrm{MG} / \mathrm{L}$ & 95 & 0.11 & 10.45 \\
\hline 7 & TURBIDITY & 4.1 & NTU & 88 & 0.09 & 7.92 \\
\hline 8 & TDS & 232 & $\mathrm{MG} / \mathrm{L}$ & 68 & 0.08 & 5.44 \\
\hline & & & & & OVI & $\mathrm{I}=74.38$ \\
\hline
\end{tabular}

Table 7: Calculation and Results of Water Quality Index (WQI)

\begin{tabular}{|c|c|c|c|c|c|c|}
\hline \multicolumn{7}{|c|}{ Station 7- Eroor Bridge Summer 2013 } \\
\hline $\begin{array}{c}\text { SI } \\
\text { No }\end{array}$ & Parame ters & Results & Units & Q Value & $\begin{array}{c}\text { Weighing } \\
\text { Factor( W) }\end{array}$ & $\begin{array}{c}\text { Sub Total } \\
(\mathbf{Q X W})\end{array}$ \\
\hline 1 & DO & 92.3 & \% SATURATION & 97 & 0.19 & 18.43 \\
\hline 2 & FC & 18.2 & MPN/100 ML & 64 & 0.18 & 11.52 \\
\hline 3 & PH & 8.3 & PH UNITS & 73 & 0.12 & 8.76 \\
\hline 4 & BOD & 2.38 & MG/L & 72 & 0.12 & 8.64 \\
\hline 5 & P & 1.4 & NTU & 33 & 0.11 & 3.63 \\
\hline 6 & N & 1.83 & MG/L & 95 & 0.11 & 10.45 \\
\hline 7 & TURBIDITY & 4.2 & NTU & 88 & 0.09 & 7.92 \\
\hline 8 & TDS & 276 & MG/L & 63 & 0.08 & 5.04 \\
\hline & & & & & & OVER ALL WQI $=74.39$ \\
\hline
\end{tabular}

Table 8: Calculation and Results of Water Quality Index (WQI)

\begin{tabular}{|c|c|c|c|c|c|l|}
\hline \multicolumn{7}{|c|}{ Station 8- Fact Cochin Discharge Out Let Summer 2013 } \\
\hline SI No & Parameters & Results & Units & Q Value & $\begin{array}{c}\text { Weighing } \\
\text { Factor( W) }\end{array}$ & Sub Total (QXW) \\
\hline 1 & DO & 92.1 & \% SATURATION & 97 & 0.19 & 18.43 \\
\hline 2 & FC & 18.35 & MPN/100 ML & 64 & 0.18 & 11.52 \\
\hline 3 & PH & 8.4 & PH UNITS & 70 & 0.12 & 8.4 \\
\hline 4 & BOD & 2.42 & MG/L & 72 & 0.12 & 8.64 \\
\hline 5 & P & 1.42 & NTU & 32 & 0.11 & 3.52 \\
\hline 6 & N & 1.85 & MG/L & 95 & 0.11 & 10.45 \\
\hline 7 & TURIDITY & 4.7 & NTU & 87 & 0.09 & 7.83 \\
\hline 8 & TDS & 290 & MG/L & 61 & 0.08 & 4.88 \\
\hline & & & & & OVER ALL WQI $=73.67$ \\
\hline
\end{tabular}

Table 9: Calculation and Results of Water Quality Index (WQI)

\begin{tabular}{|c|c|c|c|c|c|c|}
\hline \multicolumn{7}{|c|}{ Station 9- Fact B arge Jetty Summer 2013 } \\
\hline $\begin{array}{c}\text { SI } \\
\text { No }\end{array}$ & Parameters & Results & Units & Q Value & $\begin{array}{c}\text { Weighing } \\
\text { Factor }(\mathbf{W})\end{array}$ & Sub Total (QXW) \\
\hline 1 & DO & 92 & \% SATURATION & 97 & 0.19 & 18.43 \\
\hline 2 & FC & 18.5 & MPN/100 ML & 64 & 0.18 & 11.52 \\
\hline 3 & PH & 8.5 & PH UNITS & 66 & 0.12 & 7.92 \\
\hline 4 & BOD & 3.48 & MG/L & 64 & 0.12 & 7.68 \\
\hline 5 & P & 1.45 & NTU & 32 & 0.11 & 3.52 \\
\hline 6 & N & 1.87 & MG/L & 95 & 0.11 & 10.45 \\
\hline 7 & TURIDITY & 11.2 & NTU & 74 & 0.09 & 6.66 \\
\hline 8 & TDS & 2226 & MG/L & 20 & 0.08 & 1.6 \\
\hline & & & & & OVER ALL WQI=67.78 \\
\hline
\end{tabular}


Table 1: Calculation and Results of Water Quality Index (WQI

\begin{tabular}{|c|c|c|c|c|c|c|}
\hline \multicolumn{7}{|c|}{ Station 1- Cochin Bar Mouth Rainy Season 2013 } \\
\hline SI No & Parameters & Results & Units & Q Value & $\begin{array}{c}\text { Weighing } \\
\text { Factor( W) }\end{array}$ & Sub Total (QXW) \\
\hline 1 & DO & 98.9 & \% SATURATION & 99 & 0.19 & 18.81 \\
\hline 2 & FC & 6.9 & MPN/100 ML & 76 & 0.18 & 13.68 \\
\hline 3 & PH & 6.2 & PH UNITS & 60 & 0.12 & 7.2 \\
\hline 4 & BOD & 0.82 & MG/L & 96 & 0.12 & 11.52 \\
\hline 5 & P & 0.03 & NTU & 99 & 0.11 & 10.89 \\
\hline 6 & N & 1.02 & MG/L & 96 & 0.11 & 10.56 \\
\hline 7 & TURIDITY & 2.12 & NTU & 93 & 0.09 & 8.37 \\
\hline 8 & TDS & 145 & MG/L & 79 & 0.08 & 6.32 \\
\hline
\end{tabular}

Table 2: Calculation and Results of Water Quality Index (WQI)

\begin{tabular}{|c|c|c|c|c|c|c|}
\hline \multicolumn{7}{|c|}{ Station 2- Oil Tanker Berth Rainy Season 2013 } \\
\hline $\begin{array}{c}\text { SI } \\
\text { No }\end{array}$ & Parameters & Results & Units & Q Value & $\begin{array}{c}\text { Weighing } \\
\text { Factor( W) }\end{array}$ & Sub Total (QXW) \\
\hline 1 & DO & 98.02 & \% SATURATION & 99 & 0.19 & 18.81 \\
\hline 2 & FC & 6.3 & MPN/100 ML & 77 & 0.18 & 13.86 \\
\hline 3 & PH & 6.6 & PH UNITS & 75 & 0.12 & 9 \\
\hline 4 & BOD & 0.87 & MG/L & 96 & 0.12 & 11.52 \\
\hline 5 & P & 0.035 & NTU & 99 & 0.11 & 10.89 \\
\hline 6 & N & 1.08 & MG/L & 96 & 0.11 & 10.56 \\
\hline 7 & TURBDITY & 2.3 & NTU & 92 & 0.09 & 8.28 \\
\hline 8 & TDS & 146 & MG/L & 79 & 0.08 & 6.32 \\
\hline & & & & & OVER ALL WQI=89.24 \\
\hline
\end{tabular}

Table 3: Calculation and Results of Water Quality Index (WQI)

\begin{tabular}{|c|c|c|c|c|c|c|}
\hline \multicolumn{7}{|c|}{ Station 3- Thoppumpady Fishing Harbour Rainy Season 2013 } \\
\hline $\begin{array}{c}\text { SI } \\
\text { No }\end{array}$ & Parameters & Results & Units & Q Value & $\begin{array}{c}\text { Weighing } \\
\text { Factor(W) }\end{array}$ & Sub Total(QXW) \\
\hline 1 & DO & 97.2 & \% SATURATION & 99 & 0.19 & 18.81 \\
\hline 2 & FC & 8.2 & MPN/100 ML & 74 & 0.18 & 13.32 \\
\hline 3 & PH & 6.9 & PH UNITS & 86 & 0.12 & 10.32 \\
\hline 4 & BOD & 1 & MG/L & 95 & 0.12 & 11.4 \\
\hline 5 & P & 0.093 & NTU & 96 & 0.11 & 10.56 \\
\hline 6 & N & 1.2 & MG/L & 96 & 0.11 & 8.56 \\
\hline 7 & TURIDITY & 1.65 & NTU & 94 & 0.09 & 6.32 \\
\hline 8 & TDS & 148 & MG/L & 79 & 0.08 & OVER ALL WQI=89.75 \\
\hline
\end{tabular}

Table 4: Calculation and Results of Water Quality Index (WQI)

\begin{tabular}{|c|c|c|c|c|c|c|}
\hline \multicolumn{7}{|c|}{ Station 4- The vara Ferry Point Rainy Season 2013 } \\
\hline SI No & Parame ters & Results & Units & Q Value & $\begin{array}{c}\text { Weighing } \\
\text { Factor( W) }\end{array}$ & Sub Total(QXW) \\
\hline 1 & DO & 96.09 & $\%$ SATURATION & 99 & 0.19 & 18.81 \\
\hline 2 & FC & 12 & MPN/100 ML & 69 & 0.18 & 12.42 \\
\hline 3 & PH & 6.9 & PH UNITS & 86 & 0.12 & 10.32 \\
\hline 4 & BOD & 1.19 & MG/L & 93 & 0.12 & 11.16 \\
\hline 5 & P & 1.02 & NTU & 40 & 0.11 & 4.4 \\
\hline 6 & N & 1.6 & MG/L & 95 & 0.11 & 10.45 \\
\hline 7 & TURIDITY & 1.75 & NTU & 94 & 0.09 & 8.46 \\
\hline 8 & TDS & 147 & MG/L & 79 & 0.08 & 6.32 \\
\hline & & & & & OVER ALL WQI=82.34 \\
\hline
\end{tabular}


Table 5: Calculation and Results of Water Quality Index (WQI)

\begin{tabular}{|c|c|c|c|c|c|c|}
\hline \multicolumn{7}{|c|}{ Station 5- Thykoodam Nh Bridge Rainy Season 2013 } \\
\hline $\begin{array}{c}\text { SI } \\
\text { No }\end{array}$ & Parameters & Results & Units & Q Value & $\begin{array}{c}\text { Weighing Factor } \\
\text { (W) }\end{array}$ & Sub Total(QXW) \\
\hline 1 & DO & 96 & \% SATURATION & 99 & 0.19 & 18.81 \\
\hline 2 & FC & 13.4 & MPN/100 ML & 68 & 0.18 & 12.24 \\
\hline 3 & PH & 6.8 & PH UNITS & 83 & 0.12 & 9.96 \\
\hline 4 & BOD & 1.01 & MG/L & 95 & 0.12 & 11.4 \\
\hline 5 & P & 1.6 & NTU & 30 & 0.11 & 3.3 \\
\hline 6 & N & 1.83 & MG/L & 95 & 0.11 & 0.45 \\
\hline 7 & TURIDITY & 1.83 & NTU & 94 & 0.09 & 8.46 \\
\hline 8 & TDS & 149 & MG/L & 79 & 0.08 & 6.32 \\
\hline & & & & & OVER ALL WQI=80.94 \\
\hline
\end{tabular}

Table 6: Calculation and Results of Water Quality Index (WQI)

\begin{tabular}{|c|c|c|c|c|c|c|}
\hline \multicolumn{7}{|c|}{ Station 6- Kaniyampuzha RI wy Bridge Rainy Season 2013 } \\
\hline $\begin{array}{c}\text { SI } \\
\text { No }\end{array}$ & Parame ters & Results & Units & Q Value & $\begin{array}{c}\text { Weighing } \\
\text { Factor( W) }\end{array}$ & Sub Total(QXW) \\
\hline 1 & DO & 95.2 & \% SATURATION & 98 & 0.19 & 18.62 \\
\hline 2 & FC & 18.3 & MPN/100 ML & 64 & 0.18 & 11.52 \\
\hline 3 & PH & 7.6 & PH UNITS & 92 & 0.12 & 11.04 \\
\hline 4 & BOD & 2.12 & MG/L & 78 & 0.12 & 9.36 \\
\hline 5 & P & 1.18 & NTU & 36 & 0.11 & 3.96 \\
\hline 6 & N & 1.42 & MG/L & 96 & 0.11 & 10.56 \\
\hline 7 & TURBDITY & 1.86 & NTU & 93 & 0.09 & 8.37 \\
\hline 8 & TDS & 148 & MG/L & 79 & 0.08 & 6.32 \\
\hline & & & & & OVER ALL WQI=79.75 \\
\hline
\end{tabular}

Table 7: Calculation and Results of Water Quality Index (WQI)

\begin{tabular}{|c|c|c|c|c|c|c|}
\hline \multicolumn{7}{|c|}{ Station 7- Eroor Bridge Rainy Season 2013 } \\
\hline $\begin{array}{c}\text { SI } \\
\text { No }\end{array}$ & Parameters & Results & Units & Q Value & $\begin{array}{c}\text { Weighing } \\
\text { Factor }(\mathbf{W})\end{array}$ & Sub Total (QXW) \\
\hline 1 & DO & 92 & \% SATURATION & 97 & 0.19 & 18.43 \\
\hline 2 & FC & 17.8 & MPN/100 ML & 65 & 0.18 & 11.7 \\
\hline 3 & PH & 7.8 & PH UNITS & 90 & 0.12 & 10.8 \\
\hline 4 & BOD & 2.17 & MG/L & 77 & 0.12 & 9.24 \\
\hline 5 & P & 1.27 & NTU & 35 & 0.11 & 3.85 \\
\hline 6 & N & 1.62 & MG/L & 95 & 0.11 & 10.45 \\
\hline 7 & TURIDITY & 1.85 & NTU & 93 & 0.09 & 8.37 \\
\hline 8 & TDS & 148 & MG/L & 79 & 0.08 & 6.32 \\
\hline & & & & & OVER ALL WQI=79.16 \\
\hline
\end{tabular}

Table 8: Calculation and Results of Water Quality Index (WQI)

\begin{tabular}{|c|c|c|c|c|c|c|}
\hline \multicolumn{7}{|c|}{ Station 8- Fact Cochin Discharge Out Let Rainy Season 2013 } \\
\hline $\begin{array}{c}\text { SI } \\
\text { No }\end{array}$ & Parameters & Results & Units & Q Value & $\begin{array}{c}\text { Weighing } \\
\text { Factor( W) }\end{array}$ & Sub Total(QXW) \\
\hline 1 & DO & 92 & \% SATURATION & 97 & 0.19 & 18.43 \\
\hline 2 & FC & 17.9 & MPN/100 ML & 65 & 0.18 & 11.7 \\
\hline 3 & PH & 7.9 & PH UNITS & 87 & 0.12 & 10.44 \\
\hline 4 & BOD & 2.17 & MG/L & 77 & 0.12 & 9.24 \\
\hline 5 & P & 1.28 & NTU & 34 & 0.11 & 3.74 \\
\hline 6 & N & 1.72 & MG/L & 95 & 0.11 & 10.45 \\
\hline 7 & TURIDITY & 1.88 & NTU & 93 & 0.09 & 8.37 \\
\hline 8 & TDS & 152 & MG/L & 79 & 0.08 & 6.32 \\
\hline & & & & & OVER ALL WQI=78.69 \\
\hline
\end{tabular}


Table 9: Calculation and Results of Water Quality Index (WQI)

\begin{tabular}{|c|c|c|c|c|c|c|}
\hline \multicolumn{7}{|c|}{ Station 9- Fact B arge Jetty- Rainy Season 2013 } \\
\hline $\begin{array}{c}\text { SI } \\
\text { No }\end{array}$ & Parameters & Results & Units & $\begin{array}{c}\text { Q } \\
\text { Value }\end{array}$ & $\begin{array}{c}\text { Weighing } \\
\text { Factor( W) }\end{array}$ & Sub Total(QXW) \\
\hline 1 & DO & 91.5 & \% SATURATION & 96 & 0.19 & 18.24 \\
\hline 2 & FC & 18.2 & MPN/100 ML & 64 & 0.18 & 11.52 \\
\hline 3 & PH & 8.2 & PH UNITS & 77 & 0.12 & 9.24 \\
\hline 4 & BOD & 3.2 & MG/L & 66 & 0.12 & 7.92 \\
\hline 5 & P & 1.32 & NTU & 34 & 0.11 & 3.74 \\
\hline 6 & N & 1.72 & MG/L & 95 & 0.11 & 10.45 \\
\hline 7 & TURIDITY & 11.1 & NTU & 74 & 0.09 & 6.66 \\
\hline 8 & TDS & 258 & MG/L & 65 & 0.08 & 5.2 \\
\hline & & & & & OVER ALL WQI=72.97 \\
\hline
\end{tabular}

\section{REFERENCES}

1. APHA (1995). Standard methods for the examination of water and wastewater, $19^{\text {th }}$ (ed) p. 1467. Washington, DC: American Public Association. Options, Current Sci., 86(9): 1216-24

2. Chanda, D, K., (1999).Hydrology Journal, 7(5), 431-439

3. Claes, M., (1997), Comaprison Study on river quality, Sci, Tot, Env.,207, 141-148

4. Gupta, S.K. \& Deshpande, R.D.(2004). Water for India in 2050: first-order assessment of available Options.Current Sci. 86(9),1216-24

5. Huge Ellis, J. (1987). Stochastic water quality optimization using imbedded chance constra ints. Water Resource Research, 123(1), 2227-2238

6. Jammel,A. (1998). Physico-chemical studies in Vyakondan channel water of Cauvery. Poll. Res., 17(2), 111-114

7. Joy, C.M,.,Balakrishnan \&Ammini Joseph,(1990). Effect of Industrial discharges on the ecology of Phyto Plankton on Plankton Production in the River Periyar(India).wat.res, 24,787-796

8. Magudeswaran, P.N (2004). Water Quality Assessment of Noyyal River. Bharathiyar University, Coimbatore.

9. Majid, M.A.,\& Sharma, S.K. (1999). A study of the water quality parameter of the Karnaphuly River.J.Ban Chem Soc, $12(1), 17-24$

10. Qram, B. (2010) - Water Quality Index: Monitoring the quality of surface waters. Retrieved from http://www. water reseach.net/waterqualityindex.htm.

11. Sunitha Hooda \&Sumanjee Kaur.(1999). Laboratory Manual for Environmental Chemistry. S Chand \& Company Limited, Ram Nagar, New Delhi.

12. Thomann,\&.Mueller. (1987). Princip les of water quality modeling and control. Harper and Row. 